\title{
The impact of transient and persistent acute kidney injury on short-term outcomes in very elderly patients
}

\author{
Qinglin $\mathrm{Li}^{1}$ \\ Meng Zhao ${ }^{2}$ \\ Xiaodan Wang' \\ 'Department of Geriatric \\ Nephrology, ${ }^{2}$ Department of Clinical \\ Data Repository, Chinese PLA \\ General Hospital, Beijing, People's \\ Republic of China
}

\author{
This article was published in the following Dove Press journal: \\ Clinical Interventions in Aging \\ 28 June 2017 \\ Number of times this article has been viewed
}

Objectives: Acute kidney injury (AKI) is a common complication in elderly patients and is associated with poor outcomes. However, the effect of transient and persistent geriatric AKI on short-term mortality is unclear. We aimed to study the incidence, clinical characteristics, and prognostic impact of transient and persistent AKI in such patients.

Methods: We retrospectively enrolled very elderly patients ( $\geq 75$ years) from the geriatric department of the Chinese PLA General Hospital between 2007 and 2015. AKI was defined according to the 2012 Kidney Disease: Improving Global Outcomes criteria. AKI patients were divided into transient or persistent AKI groups based on their renal function at 3 days post-AKI. Renal function recovery was defined as a return to the baseline serum creatinine ( $\mathrm{SCr}$ ) levels. Results: In total, 668 geriatric patients (39.0\%) experienced AKI, and 652 satisfied the inclusion criteria. Of these 652 patients, 270 (41.4\%) had transient AKI, and $382(58.6 \%)$ had persistent AKI. The 90-day mortality was $5.9 \%$ in patients with transient AKI and $53.1 \%$ in patients with persistent AKI. Multivariate analysis revealed that low hemoglobin levels (odds ratio $[\mathrm{OR}]=0.989 ; 95 \% \mathrm{CI}: 0.980-0.999 ; P=0.025)$, low mean aortic pressure $(\mathrm{OR}=0.985$; 95\% CI: 0.971-1.000; $P=0.043)$, peak $\mathrm{SCr}(\mathrm{OR}=1.020 ; 95 \% \mathrm{CI}: 1.015-1.026 ; P<0.001)$ levels, high uric acid $(\mathrm{OR}=1.002 ; 95 \% \mathrm{CI}: 1.000-1.003 ; P=0.040)$ levels, high blood urea nitrogen ( $\mathrm{OR}=1.028 ; 95 \% \mathrm{CI}: 1.000-1.056 ; P=0.047)$ levels, and mechanical ventilation requirements ( $\mathrm{OR}=1.610 ; 95 \% \mathrm{CI}: 1.012-2.562 ; P=0.044)$ were associated with persistent $\mathrm{AKI}$. Persistent AKI (hazard ratio [HR] $=5.741 ; 95 \% \mathrm{CI}: 3.356-9.822 ; P<0.001$ ) and more severe AKI stages (stage 2 : $\mathrm{HR}=3.363 ; 95 \% \mathrm{CI}: 1.973-5.732 ; P<0.001$ and stage 3 : $\mathrm{HR}=4.741$; 95\% CI: $2.807-8.008 ; P<0.001)$ were associated with 90-day mortality.

Conclusion: AKI is common in very elderly patients, with transient renal injury representing close to $42 \%$ of all cases of geriatric AKI. More frequent SCr measurements may be helpful for the early diagnosis of transient geriatric AKI. Persistent geriatric AKI is independently associated with a significantly higher risk of 90-day mortality.

Keywords: acute kidney injury, very elderly, short-term mortality, transient and persistent, risk factors

\section{Introduction}

Acute kidney injury (AKI), which occurs in $23 \%-40 \%$ of elderly patients, ${ }^{1-5}$ is associated with an increased risk of both chronic kidney disease (CKD) and death. ${ }^{6-9}$ The key to improving the prognosis of AKI is early diagnosis and early intervention. Nonetheless, AKI diagnosis currently still relies on an increase in serum creatinine ( $\mathrm{SCr}$ ) or a decrease in urine output, which are late markers of impaired renal function. ${ }^{10,11}$ Delayed AKI diagnosis is probably one of the main reasons for its high morbidity and mortality. ${ }^{12}$
Correspondence: Xiaodan Wang Department of Health Care, Chinese PLA General Hospital, 28 Fuxing Road, 100853 Beijing, People's Republic of China Email xdwangIII@hotmail.com 
It should be noted, however, that only a small percentage of AKI patients undergo severe AKI or need dialysis, whereas the majority of AKI patients suffer from milder degrees of renal injury. ${ }^{13,14}$ The early recognition of AKI is important for preventing or minimizing the associated adverse outcomes. ${ }^{11}$ However, unfortunately, most general units routinely measure $\mathrm{SCr}$ only once or twice a week. This practice may also be a factor in the delay of AKI diagnosis, especially in situations where small changes in $\mathrm{SCr}$ occur in elderly people who lack baseline $\mathrm{SCr}$ measurements. It is also possible that such impaired renal injury occasionally may be very transient. Therefore, the SCr-based diagnosis of very transient AKI would be missed, underestimating the real incidence of AKI in the very elderly population..$^{15,16}$

The clinical implications of transient and persistent AKI in very elderly patients are unknown. Therefore, the goals of the present study were to 1) compare the rates of transient and persistent AKI from different AKI stages; 2) address the key clinical differences between transient and persistent AKI; and 3) examine the effect of transient and persistent AKI on short-term mortality.

\section{Materials and methods}

This study was conducted at the Geriatric Department of the Chinese PLA General Hospital. The study design was approved by the Clinical Ethics Committee of the Chinese PLA General Hospital, and each patient provided informed written consent. We included all hospitalizations of very elderly patients ( $\geq 75$ years of age) who were admitted between January 2007 and December 2015. All admissions were screened and evaluated for AKI, categorizing them according to the Kidney Disease Improving Global Outcomes (KDIGO) criteria. ${ }^{10}$ Exclusion criteria included patients who had been previously diagnosed with $\mathrm{CKD},{ }^{17}$ stayed in the hospital for $<2$ days, lacked at least $2 \mathrm{SCr}$ measurements during hospitalization, and had a missing or incomplete medical history.

\section{Definitions}

We used the current $2012 \mathrm{KDIGO}$ definition of AKI as the major screening criteria, ${ }^{10}$ but urine output criteria were not applied. Baseline estimated glomerular filtration rates (eGFRs) were calculated according to the Chronic Kidney Disease Epidemiology Collaboration. ${ }^{18}$ For baseline SCr, we used the most recent stable measurement during the previous 3 months. ${ }^{4}$ Peak SCr was the highest $\mathrm{SCr}$ reached during the episode. "Transient AKI" was defined as a return to baseline SCr within 3 days post-AKI and "Persistent AKI" was defined as renal dysfunction without recovery within 3 days. ${ }^{19,20}$

\section{Statistical analysis}

Continuous variables are presented as the mean $\pm \mathrm{SD}$, or medians (with 25th and 75th percentiles), depending on the variable distribution. Categorical variables are presented as numbers or percentages. Between-group comparisons were made using Student's $t$-test or Mann-Whitney $U$-test for continuous variables and with Pearson's chi-squared or Fisher's exact tests for categorical variables. Logistic regression analyses were performed to identify the covariates associated with persistent AKI. Survival curves were estimated by the Kaplan-Meier product-limit method and compared by the Mantel (log-rank) test. Prognostic survival factors were identified using the Cox proportional hazards regression model. A $P$-value $<0.05$ indicated statistical significance. Statistical analyses were performed using SPSS version 17.0 for Windows (SPSS Inc., Chicago, IL, USA).

\section{Results \\ Study population}

Between January 2007 and December 2015, a total of 3,464 very elderly patients were admitted to the Geriatric Department, and 668 developed AKI during hospitalization. Of these, 10 were excluded for hospital stays $<48 \mathrm{~h}$, and six for missing data required for this study, resulting in $652 \mathrm{AKI}$ patients who were suitable for the final evaluation, including $382(58.6 \%)$ patients with persistent AKI and 270 (41.4\%) patients with transient AKI. The overall 90-day mortality was $33.6 \%$ (219/652). The study flow chart is presented as Figure 1.

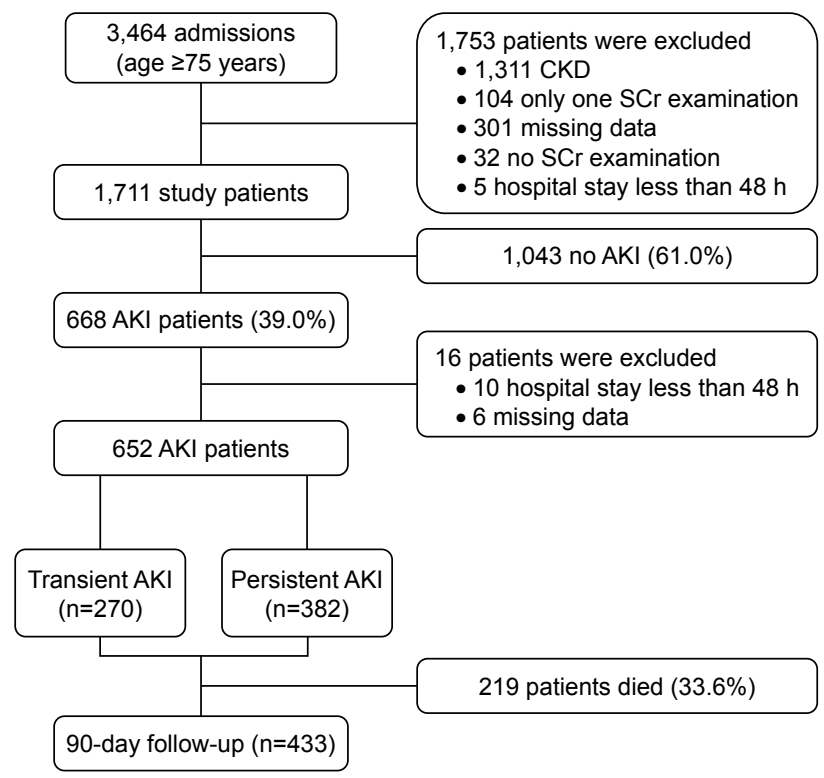

Figure I Flow chart of patient inclusion and exclusion. Abbreviations: AKI, acute kidney injury; CKD, chronic kidney disease; SCr, serum creatinine. 


\section{AKI}

Baseline characteristics for all study participants are reported in Table 1 . There were 652 elderly AKI patients with a median age of 87 (84-91), the majority $(623,95.6 \%)$ of whom were male. Among these 652 patients, $308(47.2 \%)$ had stage 1 AKI, 164 (25.2\%) had stage 2 AKI, 171 (26.2\%) had stage 3 AKI without the need for acute dialysis, and $9(1.4 \%)$ needed dialysis. Oliguria was observed in 35 cases (5.4\%).

\section{Clinical characteristics associated with transient and persistent AKI}

As shown in Table 1, comparison of the elderly patients with transient and persistent AKI indicated no significant differences in age (median age: 87 vs 87 years, $P=0.400$ ) or body mass index (BMI) $\left(23.3 \pm 3.5 \mathrm{~kg} / \mathrm{m}^{2}\right.$ vs $\left.22.9 \pm 3.0 \mathrm{~kg} / \mathrm{m}^{2}, P=0.149\right)$. Similarly, no significant differences were found for preexisting comorbidities (coronary disease $P=0.432$, hypertension $P=0.977$, COPD $P=0.864$, and diabetes mellitus $P=0.987$ ) or in baseline renal function (baseline $\mathrm{SCr} P=0.853$ and eGFR $P=0.840$ ). Patients with persistent AKI were significantly more likely to be male $(97.1 \%$ vs $93.3 \%, P=0.021)$. Patients with persistent AKI were more frequently treated with mechanical ventilation ( $\mathrm{MV}, 49.0 \%$ vs $19.6 \%, P<0.001$ ), needed dialysis ( $2.4 \%$ vs $0 \%, P=0.028)$, and suffered from low mean arterial pressure (MAP, $76 \pm 14 \mathrm{mmHg}$ vs $82 \pm 14 \mathrm{mmHg}, P<0.001$ ) and oliguria $(7.6 \%$ vs $2.2 \%, P=0.003)$. They also had

Table I Characteristics of patients with transient and persistent AKI

\begin{tabular}{|c|c|c|c|c|}
\hline Characteristic & $\begin{array}{l}\text { AKI patients } \\
(n=652)\end{array}$ & $\begin{array}{l}\text { Transient AKI } \\
n=270(41.4 \%)\end{array}$ & $\begin{array}{l}\text { Persistent AKI } \\
n=382(58.6 \%)\end{array}$ & P-value \\
\hline Age (years) & 87 (84-9I) & 87 (83.8-91) & 87 (84-9I) & 0.400 \\
\hline Male & $623(95.6)$ & $252(93.3)$ & $37 I(97 . I)$ & 0.021 \\
\hline $\mathrm{BMI}\left(\mathrm{kg} / \mathrm{m}^{2}\right)$ & $23.1 \pm 3.2$ & $23.3 \pm 3.5$ & $22.9 \pm 3.0$ & 0.149 \\
\hline \multicolumn{5}{|l|}{ Comorbidity } \\
\hline Coronary disease & 505 (77.5) & 205 (75.9) & $300(78.5)$ & 0.432 \\
\hline Hypertension & $485(74.4)$ & $201(74.4)$ & $284(74.3)$ & 0.977 \\
\hline COPD & $454(69.6)$ & $189(70.0)$ & $265(69.4)$ & 0.864 \\
\hline Diabetes & $234(35.8)$ & $97(35.9)$ & 137 (35.9) & 0.987 \\
\hline Baseline SCr $(\mu \mathrm{mol} / \mathrm{L})$ & $73.0(61.0-84.0)$ & $74.0(63.0-82.0)$ & $72.0(60.0-85.3)$ & 0.853 \\
\hline Baseline eGFR $\left(\mathrm{mL} / \mathrm{min} / \mathrm{I} .73 \mathrm{~m}^{2}\right)$ & $78.4(71.2-85.0)$ & $78.4(72.7-84.5)$ & $78.4(70.2-85.4)$ & 0.840 \\
\hline \multicolumn{5}{|l|}{ Etiology of $\mathrm{AKI}$} \\
\hline Infections & $259(39.7)$ & $100(37.0)$ & $159(4 \mid .6)$ & 0.238 \\
\hline Hypovolemia & 155 (23.8) & $55(20.4)$ & $100(26.2)$ & 0.086 \\
\hline Cardiovascular events & $103(15.8)$ & $47(17.4)$ & $56(14.7)$ & 0.343 \\
\hline Nephrotoxicity & $78(12.0)$ & $36(13.3)$ & $42(11.0)$ & 0.365 \\
\hline Surgery & $45(6.9)$ & $25(9.3)$ & $20(5.2)$ & 0.046 \\
\hline Others & $12(1.8)$ & $7(2.6)$ & $5(1.3)$ & 0.365 \\
\hline \multicolumn{5}{|l|}{ Parameter at the time of $\mathrm{AKI}$ diagnosis } \\
\hline MAP $(\mathrm{mmHg})$ & $78 \pm 14$ & $82 \pm 14$ & $76 \pm 14$ & $<0.001$ \\
\hline Oliguria & $35(5.4)$ & $6(2.2)$ & $29(7.6)$ & 0.003 \\
\hline $\mathrm{MV}$ & $240(36.8)$ & $53(19.6)$ & $187(49.0)$ & $<0.001$ \\
\hline \multicolumn{5}{|c|}{ Laboratory results at the time of $\mathrm{AKI}$ diagnosis } \\
\hline $\mathrm{SCr}(\mu \mathrm{mol} / \mathrm{L})$ & $|3| .4(|| 7.5-\mid 47.0)$ & $122.0(|1| .0-136.0)$ & $138.0(\mid 25.0-156.6)$ & $<0.001$ \\
\hline Peak SCr $(\mu \mathrm{mol} / \mathrm{L})$ & I 43.7 ( I 24.0-200.0) & $124.2(113.5-139.1)$ & $174.3(\mid 40.5-264.9)$ & $<0.001$ \\
\hline BUN (mmol/L) & $12.6(8.8-20.9)$ & $10.1(7.5-14.7)$ & $16.2(10.0-26.7)$ & $<0.001$ \\
\hline Uric acid (mmol/L) & $364.9(290.0-467.0)$ & $338.9(275.6-421.8)$ & $393.0(307.2-499.1)$ & $<0.001$ \\
\hline Prealbumin $(g / L)$ & $181.0(\mid 40.0-231.0)$ & $197(150-234)$ & $168(133-230)$ & $<0.001$ \\
\hline Albumin $(g / L)$ & $34.3 \pm 5.5$ & $36.0 \pm 5.2$ & $33.1 \pm 5.4$ & $<0.001$ \\
\hline Magnesium (mmol/L) & $0.9(0.8-1.0)$ & $0.9(0.8-1.0)$ & $0.9(0.8-1.0)$ & 0.002 \\
\hline Calcium (mmol/L) & $2.2(2.1-2.3)$ & $2.2(2.1-2.3)$ & $2.2(2.1-2.4)$ & 0.905 \\
\hline Phosphate (mmol/L) & $1.2(1.0-1.5)$ & $1.2(0.9-1.4)$ & $1.2(1.0-1.5)$ & 0.014 \\
\hline Hemoglobin $(g / L)$ & $112 \pm 23$ & $119 \pm 20$ & $108 \pm 23$ & $<0.001$ \\
\hline \multicolumn{5}{|l|}{ AKI stage } \\
\hline 1 & 308 (47.2) & 190 (70.4) & I 18 (30.9) & $<0.001$ \\
\hline 2 & $164(25.2)$ & $63(23.3)$ & 101 (26.4) & \\
\hline 3 & $180(27.6)$ & $17(6.3)$ & $163(42.7)$ & \\
\hline \multicolumn{5}{|l|}{ Outcome } \\
\hline Dialysis & $9(1.4)$ & 0 & $9(2.4)$ & 0.028 \\
\hline Mortality & $219(33.6)$ & $16(5.9)$ & $203(53.1)$ & $<0.001$ \\
\hline
\end{tabular}

Note: Values are $\mathrm{n}(\%)$, mean $\pm S D$, or median (interquartile range).

Abbreviations: AKI, acute kidney injury; BMI, body mass index; BUN, blood urea nitrogen; eGFR, estimated glomerular fitration rate; MAP, mean aortic pressure (I mmHg, $0.133 \mathrm{kPa}$ ); $\mathrm{MV}$, mechanical ventilation; $\mathrm{SCr}$, serum creatinine. 
significantly higher $\mathrm{SCr}(138.0$ vs $122.0 \mu \mathrm{mol} / \mathrm{L}, P<0.001)$ and peak $\operatorname{SCr}(174.3$ vs $124.2 \mu \mathrm{mol} / \mathrm{L}, P<0.001)$ levels as well as higher blood urea nitrogen (BUN) (16.2 vs $10.1 \mathrm{mmol} / \mathrm{L}$, $P<0.001)$ and uric acid (393 vs $338.9 \mu \mathrm{mol} / \mathrm{L}, P<0.001$ ) levels at the time of AKI diagnosis compared with patients with transient AKI. Low hemoglobin levels $(108 \pm 23$ vs $119 \pm 20 \mathrm{~g} / \mathrm{L}, P<0.001)$, lower prealbumin levels (168 vs $197 \mathrm{~g} / \mathrm{L}, P<0.001)$, and hypoalbuminemia $(33.1 \pm 5.4 \mathrm{vs}$ $36.0 \pm 5.2 \mathrm{~g} / \mathrm{L}, P<0.001)$ were more common in patients with persistent AKI. Patients with persistent AKI presented higher mortality rates $(53.1 \%$ vs $5.9 \%, P<0.001)$ and more frequently exhibited stage 2 and 3 AKI (26.4\% vs $23.3 \%$, $42.7 \%$ vs $6.3 \%$ ); there were fewer patients with stage 1 AKI $30.9 \%$ vs $70.4 \% ; P<0.001$; Figure 2$)$.

\section{Factors associated with persistent AKI}

Multivariate logistic regression analysis revealed that persistent AKI was significantly associated with low MAP (odds ratio [OR] $=0.985 ; 95 \%$ CI: $0.971-1.000$; $P=0.043)$, low hemoglobin levels $(\mathrm{OR}=0.989 ; 95 \% \mathrm{CI}$ : 0.980-0.999; $P=0.025)$, peak SCr levels ( $\mathrm{OR}=1.020 ; 95 \%$ CI: $1.015-1.026 ; P<0.001)$, high BUN levels ( $\mathrm{OR}=1.028 ; 95 \%$ CI: $1.000-1.056 ; P=0.047)$, high uric acid levels ( $\mathrm{OR}=1.002$; 95\% CI: $1.000-1.003 ; P=0.040)$, and a need for MV (OR =1.610; 95\% CI: 1.012-2.562; $P=0.044$; Table 2).

\section{Influence of transient or persistent AKI on patient short-term outcomes}

As shown in Table 1, the 90 -day mortality was $5.9 \%$ for patients with transient AKI and $53.1 \%$ for patients with persistent AKI $(P<0.001)$. Dialysis was necessary in $2.4 \%$ of the persistent AKI patients compared to $0 \%$ of the transient AKI $(P=0.028)$ patients. Kaplan-Meier curves showed significant differences in the 90 -day mortality between the two groups

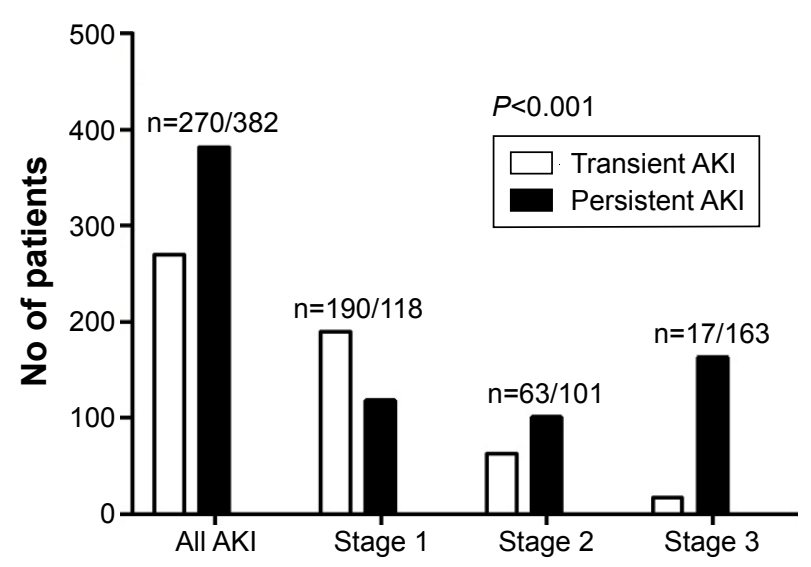

Figure 2 Transient and persistent AKI at different AKI stages in patients with AKI. Abbreviation: AKI, acute kidney injury.
Table 2 Factors associated with persistent AKI as indicated by logistic regression

\begin{tabular}{llll}
\hline Risk factor & OR & $\mathbf{9 5 \% ~ C l ~}$ & $P$-value \\
\hline MAP & 0.985 & $0.971-1.000$ & 0.043 \\
Hemoglobin & 0.989 & $0.980-0.999$ & 0.025 \\
Peak SCr level & 1.020 & $1.015-1.026$ & $<0.001$ \\
BUN level & 1.028 & $1.000-1.056$ & 0.047 \\
Uric acid & 1.002 & $1.000-1.003$ & 0.040 \\
MV & 1.610 & $1.012-2.562$ & 0.044 \\
\hline
\end{tabular}

Abbreviations: $\mathrm{AKI}$, acute kidney injury; $\mathrm{BUN}$, blood urea nitrogen; $\mathrm{Cl}$, confidence interval; MAP, mean aortic pressure $(\mathrm{I} \mathrm{mmHg}, 0.133 \mathrm{kPa})$; $\mathrm{MV}$, mechanical ventilation; $\mathrm{OR}$, odds ratio; $\mathrm{SCr}$, serum creatinine.

(log rank $P<0.001$; Figure 3). Within the AKI groups, the 90-day mortality was better in the transient AKI group than in the persistent AKI group (log rank $P<0.001$; Figure 3$)$. The separation of the curves continued throughout the follow-up period, with an increased probability of death during the follow-up with increasing degrees of AKI (Figure 4). However, in each of the AKI subgroups, the short-term mortality of patients with transient AKI was better than that of patients with persistent AKI. Only patients with transient AKI stage 3 appeared worse than patients with persistent AKI stage 1 .

Of the 652 AKI patients, the 90-day mortality was 33.6\% (219/652). Survivors were significantly more likely to have hypertension $(77.4 \%$ vs $68.5 \%, P=0.026)$. BMI $(22.2 \pm 2.8$ vs $\left.23.5 \pm 3.3 \mathrm{~kg} / \mathrm{m}^{2}, P<0.001\right)$, baseline $\mathrm{SCr}(64.0 \mathrm{vs} 78.0 \mu \mathrm{mol} / \mathrm{L}$, $P<0.001)$, and baseline eGFR $\left(83.6 \mathrm{~mL} / \mathrm{min} / 1.73 \mathrm{~m}^{2}\right.$ vs $\left.76.7 \mathrm{~mL} / \mathrm{min} / 1.73 \mathrm{~m}^{2}, P<0.001\right)$ differed significantly between those who died and survived.

As shown in Table 3, nonsurvivors were more likely to have required MV and to have had sepsis upon AKI diagnosis

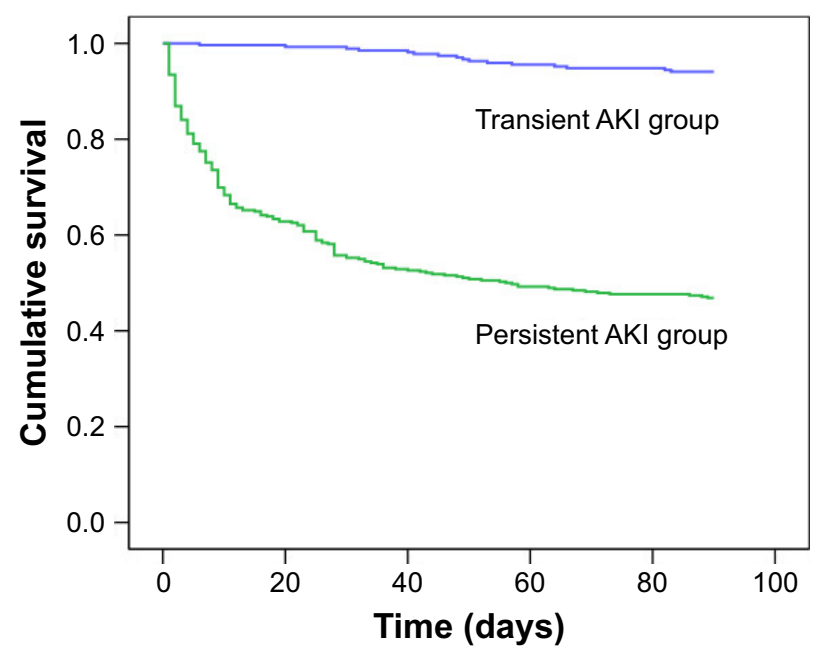

Figure 3 Kaplan-Meier survival curves according to transient and persistent AKI (log-rank test: $P<0.00 \mathrm{I}$ ).

Abbreviation: AKI, acute kidney injury. 


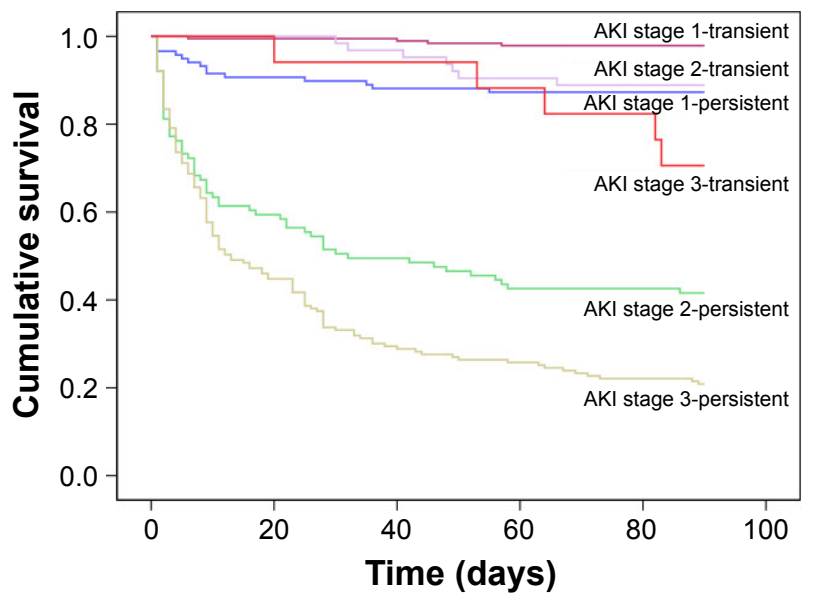

Figure 4 Kaplan-Meier survival curves according to AKI stage (log-rank test: $P<0.00$ I).

Abbreviation: AKI, acute kidney injury.

than were survivors $(65.3 \%$ vs $22.4 \%, 53 \%$ vs $33 \%$, respectively; both $P<0.001)$. Oliguria ( $11.4 \%$ vs $2.3 \%, P<0.001)$ and low MAP $(71 \pm 13$ vs $82 \pm 13 \mathrm{mmHg}, P<0.001)$ were more frequent among nonsurvivors. Likewise, lower prealbumin levels (142 vs $204 \mathrm{~g} / \mathrm{L}, P<0.001$ ), low hemoglobin levels (102 \pm 23 vs $118 \pm 20 \mathrm{~g} / \mathrm{L}, P<0.001)$, hypoalbuminemia (31.0 \pm 4.9 vs $36.0 \pm 5.0 \mathrm{~g} / \mathrm{L}, P<0.001)$, higher magnesium levels ( 0.9 vs $0.9 \mathrm{mmol} / \mathrm{L}, P=0.023$ ), and higher phosphate levels ( $1.3 \mathrm{vs} 1.2 \mathrm{mmol} / \mathrm{L}, P<0.001)$ were more frequent in those who died. As expected, the survivors had lower $\mathrm{SCr}$, BUN, uric acid, and peak $\mathrm{SCr}$ levels at the time of $\mathrm{AKI}$ diagnosis (all $P<0.001$ ). Accordingly, the prevalence of persistent AKI was significantly higher in the death group (92.7\% vs $41.3 \%, P<0.001)$.

Table 3 also shows the relationship between the AKI stage and short-term outcome; AKI severity was associated with a significantly higher 90 -day mortality $(8.7 \%$ for stage 1 patients, $30.1 \%$ for stage 2 , and $61.2 \%$ for stage 3 ). Unsurprisingly, outcomes worsened with more advanced AKI stage ( $P<0.001$ for the three stages).

In the multivariate Cox regression analysis, persistent AKI ( $\mathrm{HR}=5.741 ; 95 \% \mathrm{CI}: 3.356-9.822 ; P<0.001)$ and more severe AKI stages (stage 2 : HR $=3.363 ; 95 \%$ CI: $1.973-5.732 ; P<0.001$ and stage $3: \mathrm{HR}=4.741 ; 95 \%$ CI: 2.807-8.008; $P<0.001)$ were associated with the 90-day mortality (Table 4). The other independent risk factors for 90-day mortality included BMI, low MAP, low prealbumin levels, infection, oliguria, and BUN levels.

\section{Discussion}

We performed a single-center retrospective analysis to describe the epidemiology of AKI and its outcome associations in very elderly patients. Although older people have a high risk to develop AKI, there is no information regarding the incidence and clinical significance of transient versus persistent AKI in such patients. The present study adds interesting information by exploring the relationship between transient and persistent geriatric AKI and shortterm mortality. First, nearly $42.0 \%$ of the patients with AKI had transient AKI according to the KDIGO criteria. These patients exhibited a temporary decline of renal function and were less likely to have had oliguria and required acute dialysis. Second, geriatric patients with persistent AKI were independently associated with a higher 90-day mortality, and transient AKI was not associated with poorer shortterm outcomes. Third, MV affects an elderly patient's renal function, but does not affect short-term survival. Finally, the outcome of patients with transient AKI stage 3 was worse than that of those with persistent AKI stage 1. This finding further suggests that the distinction between transient and persistent AKI, based on full reversal within 3 days, might be a relevant end point for future studies aiming to improve the ability to predict short-term outcomes.

The first interesting finding from our study is that AKI was diagnosed in $668 / 1,711$ patients $(39.0 \%)$, and up to $42 \%$ of the AKI patients exhibited transient AKI. The higher incidence according to the KDIGO definition primarily resulted from the close monitoring of patients with mild (stage 1) AKI. We thought that this was important because, in contrast to intensive care unit patients, patients in general departments did not have routine daily $\mathrm{SCr}$ measurements because many clinicians rely on blood tests as needed and not on a daily basis. The proportion of patients with two or more SCr measurements during hospitalization ranged from $25 \%$ to $30 \%$ in previous reports. ${ }^{19,21,22}$ Even for patients with two SCr examinations in 7 days, AKI would be missed in $48 \%$ of the patients. Indeed, although the KDIGO criteria provide a 48-hour or 7-day window for diagnosis, these criteria are not commonly used in the real clinical setting. Therefore, some geriatric patients with AKI could be misclassified as not having $\mathrm{AKI}$ or, in cases of early AKI, as persistent rather than transient AKI. This could lead to an underestimation of the effect of AKI on mortality, especially in the case of transient AKI. We also divided the AKI patients into transient AKI and persistent AKI groups based on the SCr levels 3 days post-AKI. In contrast, some authors distinguish between transient and persistent AKI based on the differences between admission and discharge $\mathrm{SCr}$ values. However, admission $\mathrm{SCr}$ values may be affected by the hemodynamics or metabolic status at the time of presentation; thus, it is inappropriate 
Table 3 Comparison of clinical characteristics between elderly AKI survivors and nonsurvivors

\begin{tabular}{|c|c|c|c|c|}
\hline Characteristic & $\begin{array}{l}\text { AKI patients } \\
(n=652)\end{array}$ & $\begin{array}{l}\text { Nonsurvivors } \\
n=219(33.6 \%)\end{array}$ & $\begin{array}{l}\text { Survivors } \\
n=433(66.4 \%)\end{array}$ & $P$-value \\
\hline Age (years) & 87 (84-9I) & $88(83.8-90)$ & 87 (84-9I) & 0.444 \\
\hline Male & $623(95.6)$ & $207(94.5)$ & $416(96.1)$ & 0.258 \\
\hline BMI $\left(\mathrm{kg} / \mathrm{m}^{2}\right)$ & $23.1 \pm 3.2$ & $22.2 \pm 2.8$ & $23.5 \pm 3.3$ & $<0.001$ \\
\hline \multicolumn{5}{|l|}{ Comorbidity } \\
\hline Coronary disease & $505(77.5)$ & I7I (78.I) & $334(77.1)$ & 0.855 \\
\hline Hypertension & $485(74.4)$ & $150(68.5)$ & $335(77.4)$ & 0.026 \\
\hline COPD & $454(69.6)$ & I $47(67.1)$ & 307 (70.9) & 0.365 \\
\hline Diabetes & $234(35.8)$ & $83(37.9)$ & 151 (34.9) & 0.432 \\
\hline Baseline $\mathrm{SCr}(\mu \mathrm{mol} / \mathrm{L})$ & $73.0(61.0-84.0)$ & $64.0(54.0-75.0)$ & $78.0(66.0-86.0)$ & $<0.001$ \\
\hline Baseline eGFR $\left(\mathrm{mL} / \mathrm{min} / 1.73 \mathrm{~m}^{2}\right)$ & $78.4(71.2-85.0)$ & $83.6(76.5-90.0)$ & $76.7(69.3-81.8)$ & $<0.001$ \\
\hline \multicolumn{5}{|l|}{ Etiology of AKI } \\
\hline Infections & 259 (39.7) & $116(53.0)$ & $143(33.0)$ & $<0.001$ \\
\hline Hypovolemia & $155(23.8)$ & $53(24.2)$ & $102(23.6)$ & 0.621 \\
\hline Cardiovascular events & $103(15.8)$ & $27(12.3)$ & $76(17.6)$ & 0.088 \\
\hline Nephrotoxicity & $78(12.0)$ & $12(5.5)$ & $66(15.2)$ & 0.001 \\
\hline Surgery & $45(6.9)$ & $9(4.1)$ & $36(8.3)$ & 0.058 \\
\hline Others & $12(1.8)$ & $2(0.9)$ & $10(2.3)$ & 0.251 \\
\hline \multicolumn{5}{|l|}{ Parameter at the time of $\mathrm{AKI}$ diagnosis } \\
\hline MAP $(\mathrm{mmHg})$ & $78 \pm 14$ & $7 I \pm 13$ & $82 \pm 13$ & $<0.001$ \\
\hline Oliguria & $35(5.4)$ & $25(11.4)$ & $10(2.3)$ & $<0.001$ \\
\hline Dialysis & $9(1.4)$ & $6(27)$ & $3(0.7)$ & 0.117 \\
\hline $\mathrm{MV}$ & $240(36.8)$ & $143(65.3)$ & $97(22.4)$ & $<0.001$ \\
\hline \multicolumn{5}{|c|}{ Laboratory results at the time of $\mathrm{AKI}$ diagnosis } \\
\hline $\mathrm{SCr}(\mu \mathrm{mol} / \mathrm{L})$ & $|3| .4(|| 7.5-\mid 47.0)$ & $139.0(\mid 22.7-160.6)$ & $\mid 28.0(|| 7.0-|4| .0)$ & $<0.001$ \\
\hline Peak SCr $(\mu \mathrm{mol} / \mathrm{L})$ & I 43.7 (I24.0-200.0) & $211.7(154.3-319.7)$ & |34.9 (| |8.8-|53.2) & $<0.001$ \\
\hline BUN (mmol/L) & $12.6(8.8-20.9)$ & $21.5(14.6-32.1)$ & $10.3(7.8-15.4)$ & $<0.001$ \\
\hline Uric acid (mmol/L) & $364.9(290.0-467.0)$ & $410.1(313.5-524.1)$ & $352.0(281.0-437.6)$ & $<0.001$ \\
\hline Prealbumin $(g / L)$ & $181.0(\mid 40.0-231.0)$ & $142(\mid 10-186)$ & $204(158-256)$ & $<0.001$ \\
\hline Albumin $(g / L)$ & $34.3 \pm 5.5$ & $31.0 \pm 4.9$ & $36.0 \pm 5.0$ & $<0.001$ \\
\hline Magnesium (mmol/L) & $0.9(0.8-1.0)$ & $0.9(0.8-1.1)$ & $0.9(0.8-1.0)$ & 0.023 \\
\hline Calcium (mmol/L) & $2.2(2.1-2.3)$ & $2.2(2.0-2.3)$ & $2.2(2.1-2.4)$ & 0.120 \\
\hline Phosphate (mmol/L) & $1.2(1.0-1.5)$ & $1.3(1.0-1.6)$ & $1.2(1.0-1.4)$ & $<0.001$ \\
\hline Hemoglobin (g/L) & $112 \pm 23$ & $102 \pm 23$ & $118 \pm 20$ & $<0.001$ \\
\hline \multicolumn{5}{|l|}{ AKI stage } \\
\hline I & $308(47.2)$ & $19(8.7)$ & $289(66.7)$ & $<0.001$ \\
\hline 2 & $164(25.2)$ & $66(30.1)$ & $98(22.6)$ & \\
\hline 3 & $180(27.6)$ & $134(6 \mid .2)$ & $46(10.6)$ & \\
\hline \multicolumn{5}{|l|}{ Type of AKI } \\
\hline Transient AKI & $270(4 I .4)$ & $16(7.3)$ & $254(58.7)$ & $<0.001$ \\
\hline Persistent AKI & $382(58.6)$ & $203(92.7)$ & I 79 (4I.3) & \\
\hline
\end{tabular}

Note: Values are $\mathrm{n}(\%)$, mean $\pm \mathrm{SD}$, or median (interquartile range).

Abbreviations: AKI, acute kidney injury; BMI, body mass index; BUN, blood urea nitrogen; eGFR, estimated glomerular fitration rate; MAP, mean aortic pressure (I mmHg, $0.133 \mathrm{kPa}$ ); $\mathrm{MV}$, mechanical ventilation; $\mathrm{SCr}$, serum creatinine.

to consider this level as a baseline for renal function for study patients. In elderly patients, various chronic comorbidities and the necessity of prolonged MV can often extend hospital stays. Thus, an evaluation at hospital discharge may also not be an ideal timing.

Several studies that have assessed the prognostic impact of transient changes in renal function during hospitalization show conflicting results. ${ }^{20,23-25}$ Few studies have evaluated the prognostic impact of transient or persistent AKI in very elderly patients. It has been postulated that transient AKI is not a benign form of AKI that does not reduce mortality. In our study, $42 \%$ of hospital-acquired AKI cases were transient. Transiency did not increase the risk of mortality in patients, suggesting that transient geriatric AKI may be a benign form of AKI. In a large multicenter, retrospective study, Uchino et al examined 3,641 AKI patients using the Risk, Injury, Failure, Loss and End-stage Kidney Disease (RIFLE) definition. ${ }^{24}$ They reported that transient 
Table 4 Multivariate Cox regression analysis for 90-day mortality

\begin{tabular}{|c|c|c|c|}
\hline Risk factor & HR & $95 \% \mathrm{Cl}$ & $P$-value \\
\hline BMI & 0.939 & $0.897-0.984$ & 0.008 \\
\hline MAP & 0.969 & $0.959-0.979$ & $<0.001$ \\
\hline Prealbumin & 0.935 & $0.911-0.959$ & $<0.00$ I \\
\hline Infection & 1.410 & $1.055-1.884$ & 0.020 \\
\hline Oliguria & 1.948 & $1.266-2.998$ & 0.002 \\
\hline BUN level & 1.025 & $1.014-1.037$ & $<0.001$ \\
\hline \multicolumn{4}{|l|}{ AKI stage } \\
\hline Stage I & Reference & Reference & $<0.00$ I \\
\hline Stage 2 & 3.363 & $1.973-5.732$ & $<0.001$ \\
\hline Stage 3 & 4.741 & $2.807-8.008$ & $<0.001$ \\
\hline Persistent AKI & $5.74 I$ & $3.356-9.822$ & $<0.001$ \\
\hline
\end{tabular}

Abbreviations: $\mathrm{AKI}$, acute kidney injury; $\mathrm{BMI}$, body mass index; $\mathrm{BUN}$, blood urea nitrogen; HR, hazard ratio; MAP, mean aortic pressure, $1 \mathrm{mmHg}, 0.133 \mathrm{kPa}$.

AKI (defined as recovery of renal function to a non-AKI RIFLE category within 3 days after AKI onset) represented $32.1 \%$ of all AKI patients, that both transient and persistent AKI were independently associated with a poorer outcomes. The authors also found that patients with persistent AKI had a higher risk of death than did patients with transient AKI. Similarly, Choi et al retrospectively analyzed 2,110 patients who had been diagnosed with acute myocardial infarction, of whom 11\% developed AKI (KDIGO criteria); transient AKI (defined as normalization of SCr levels at discharge) occurred in $65 \%$ of those with AKI.$^{25}$ In this study, transient AKI was independently associated with increasing longterm mortality. In contrast, in a study by Perinel et al, which was a retrospective multicenter analysis that included 283 critically ill AKI patients (Acute Kidney Injury Network criteria), ${ }^{20}$ persistent AKI was defined as nonrecovery within 3 days, and recovery was defined as the absence of oliguria and a return to baseline $\mathrm{SCr}$ levels (or a $50 \%$ reduction). In this study, 175 patients had persistent AKI, and 108 patients had transient AKI. The authors reported no independent associations between persistent AKI and poor outcomes.

The third interesting finding is the effect of MV on elderly patients. Unlike other studies, we did not find that MV was associated with 90-day mortality, ${ }^{26}$ but was associated with persistent AKI. First, MV may affect the kidney by causing hemodynamic abnormalities and affecting renal perfusion by decreasing GFR by reducing cardiac output and stimulating hormonal and sympathetic pathways. ${ }^{27}$ Second, MV through the manipulation of permissive hypercapnia or permissive hypoxemia can lead to renal hypoperfusion, decreased GFR, and functional renal insufficiency. ${ }^{28}$ Our results also confirmed that patients with persistent AKI had a higher frequency of MV requirements and lower MAP levels compared with patients with transient AKI. The independent risk factors associated with persistent kidney injury were MV and low MAP.

The last interesting finding is in agreement with earlier studies, as we found a graded relationship between AKI severity and mortality. ${ }^{4,29,30}$ However, for each level of AKI, the outcome of patients with transient AKI stage 3 was worse than that of those with persistent AKI stage 1. This indicates that milder AKI affects patients' long-term outcomes, while severe AKI affects patients' short-term outcomes. In addition to the greater susceptibility to AKI, its diagnosis in elderly patients can be difficult or delayed, due to loss of muscle mass, and consequently lower baseline SCr level, masking an increase of its values in kidney injury pathologies, justifying the search for biomarkers of early injury, such as neutrophil gelatinase-associated lipocalin or kidney injury molecule-1.

Consistent with previous studies, we found that high uric acid was associated with persistent AKI. ${ }^{31-34}$ The plausible explanations were as follows. Uric acid has been linked to AKI via crystal-independent mechanisms, as well as crystaldependent pathways. ${ }^{32}$ High uric acid level can cause renal vasoconstriction and impair autoregulation, which results in decreased renal blood flow and GFR. ${ }^{32,33}$ Sánchez-Lozada et al reported that even a mild elevation of uric acid can induce renal vasoconstriction and persistent glomerular hypertension in rats without evidence of intratubular crystal precipitation. ${ }^{35}$ In addition, uric acid has been shown to worsen renal function via proinflammatory pathways involving chemokine expression with leukocyte infiltration, as well as proliferation of vascular smooth muscle cells and inhibition of endothelial function. . $^{33,34}$

Interestingly, previous studies reported that being male is one of the most potent risk factors for AKI. However, our multivariate analysis showed that being male was insignificant for predicting persistent AKI in this study. We speculate that this may have been caused by the small number of females in the study. In the next study, we aim to analyze the potent risk factor of being female on AKI mortality in elderly patients.

\section{Conclusion}

AKI is common in very elderly patients, and transient renal injury represents close to $42 \%$ of all cases of geriatric AKI. More frequent SCr measurements may be helpful for the early diagnosis of transient geriatric AKI. Additionally, persistent geriatric AKI is independently associated with a significantly higher risk of 90-day mortality. 


\section{Disclosure}

The authors report no conflicts of interest in this work.

\section{References}

1. Chao CT, Lin YF, Tsai HB, Wu VC, Ko WJ. Acute kidney injury network staging in geriatric postoperative acute kidney injury patients: shortcomings and improvements. J Am Coll Surg. 2013;217(2): 240-250.

2. Elmistekawy E, McDonald B, Hudson C, et al. Clinical impact of mild acute kidney injury after cardiac surgery. Ann Thorac Surg. 2014; 98(3):815-822.

3. Reents W, Hilker M, Borgermann J, et al. Acute kidney injury after on-pump or off-pump coronary artery bypass grafting in elderly patients. Ann Thorac Surg. 2014;98(1):9-14; discussion 14-15.

4. Chao CT, Tsai HB, Wu CY, et al. The severity of initial acute kidney injury at admission of geriatric patients significantly correlates with subsequent in-hospital complications. Sci Rep. 2015;5:13925.

5. Yokota LG, Sampaio BM, Rocha E, Balbi AL, Ponce D. Acute kidney injury in elderly intensive care patients from a developing country: clinical features and outcome. Int J Nephrol Renovasc Dis. 2017; 10:27-33.

6. Pannu N, James M, Hemmelgarn B, Klarenbach S; Alberta Kidney Disease Network. Association between AKI, recovery of renal function, and long-term outcomes after hospital discharge. Clin J Am Soc Nephrol. 2013;8(2):194-202.

7. Chawla LS, Kimmel PL. Acute kidney injury and chronic kidney disease: an integrated clinical syndrome. Kidney Int. 2012;82(5): 516-524.

8. Rewa O, Bagshaw SM. Acute kidney injury-epidemiology, outcomes and economics. Nat Rev Nephrol. 2014;10(4):193-207.

9. Nisula S, Kaukonen KM, Vaara ST, et al; FINNAKI Study Group. Incidence, risk factors and 90-day mortality of patients with acute kidney injury in Finnish intensive care units: the FINNAKI study. Intensive Care Med. 2013;39(3):420-428.

10. Eknoyan G, Lameire N, Eckardt K, Kasiske B. Kidney disease: improving global outcomes (KDIGO) acute kidney injury work group. KDIGO clinical practice guideline for acute kidney injury. Kidney Int Suppl. 2012;2:1-138.

11. Chronopoulos A, Cruz DN, Ronco C. Hospital-acquired acute kidney injury in the elderly. Nat Rev Nephrology. 2010;6(3):141-149.

12. Chao CT, Wu VC, Lai CF, et al. Advanced age affects the outcomepredictive power of RIFLE classification in geriatric patients with acute kidney injury. Kidney Int. 2012;82(8):920-927.

13. Thakar CV, Christianson A, Freyberg R, Almenoff P, Render ML. Incidence and outcomes of acute kidney injury in intensive care units: a Veterans Administration study. Crit Care Med. 2009;37(9): $2552-2558$

14. Liborio AB, Leite TT, Neves FM, Teles F, Bezerra CT. AKI complications in critically ill patients: association with mortality rates and RRT. Clin J Am Soc Nephrol. 2015;10(1):21-28.

15. Bagshaw SM, Uchino S, Cruz D, et al. A comparison of observed vs estimated baseline creatinine for determination of RIFLE class in patients with acute kidney injury. Nephrol Dial Transplant. 2009;24(9): 2739-2744.
16. Mehta RL. Timed and targeted therapy for acute kidney injury: a glimpse of the future. Kidney Int. 2010;77(11):947-949.

17. Levin A, Stevens PE. Summary of KDIGO 2012 CKD Guideline: behind the scenes, need for guidance, and a framework for moving forward. Kidney Int. 2014;85(1):49-61.

18. Levey AS, Stevens LA, Schmid CH, et al. A new equation to estimate glomerular filtration rate. Ann Intern Med. 2009;150(9):604-612.

19. Xu X, Nie S, Liu Z, et al. Epidemiology and clinical correlates of AKI in Chinese hospitalized adults. Clin J Am Soc Nephrol. 2015;10(9): 1510-1518.

20. Perinel S, Vincent F, Lautrette A, et al. Transient and persistent acute kidney injury and the risk of hospital mortality in critically Ill patients: results of a multicenter cohort study. Crit Care Med. 2015; 43(8):e269-e275.

21. Yang L, Xing G, Wang L, et al. Acute kidney injury in China: a crosssectional survey. Lancet. 2015;386(10002):1465-1471.

22. Lu R, Mucino-Bermejo MJ, Armignacco P, et al. Survey of acute kidney injury and related risk factors of mortality in hospitalized patients in a third-level urban hospital of Shanghai. Blood Purif. 2014; 38(2):140-148

23. Tian J, Barrantes F, Amoateng-Adjepong Y, Manthous CA. Rapid reversal of acute kidney injury and hospital outcomes: a retrospective cohort study. Am J Kidney Dis. 2009;53(6):974-981.

24. Uchino S, Bellomo R, Bagshaw SM, Goldsmith D. Transient azotaemia is associated with a high risk of death in hospitalized patients. Nephrol Dial Transplant. 2010;25(6):1833-1839.

25. Choi JS, Kim YA, Kim MJ, et al. Relation between transient or persistent acute kidney injury and long-term mortality in patients with myocardial infarction. Am J Cardiol. 2013;112(1):41-45.

26. Daher Ede F, Junior Silva GB, Vieira AP, et al. Acute kidney injury in a tropical country: a cohort study of 253 patients in an infectious diseases intensive care unit. Rev Soc Bras Med Trop. 2014;47(1):86-89.

27. Koyner JL, Murray PT. Mechanical ventilation and the kidney. Blood Purif. 2010;29(1):52-68.

28. Broden CC. Acute renal failure and mechanical ventilation: reality or myth? Crit Care Nurse. 2009;29(2):62-75; quiz 76.

29. Bagshaw SM, George C, Bellomo R; Committee ADM. Early acute kidney injury and sepsis: a multicentre evaluation. Crit Care. 2008; 12(2):R47.

30. Hobson CE, Yavas S, Segal MS, et al. Acute kidney injury is associated with increased long-term mortality after cardiothoracic surgery. Circulation. 2009;119(18):2444-2453.

31. Cheungpasitporn W, Thongprayoon C, Harrison AM, Erickson SB. Admission hyperuricemia increases the risk of acute kidney injury in hospitalized patients. Clinical kidney journal. 2016;9(1):51-56.

32. Ejaz AA, Dass B, Kambhampati G, et al. Lowering serum uric acid to prevent acute kidney injury. Med Hypotheses. 2012;78(6):796-799.

33. Lapsia V, Johnson RJ, Dass B, et al. Elevated uric acid increases the risk for acute kidney injury. Am J Med. 2012;125(3):302. e309-e317.

34. Shimada M, Dass B, Ejaz AA. Paradigm shift in the role of uric acid in acute kidney injury. Semin Nephrol. 2011;31(5):453-458.

35. Sanchez-Lozada LG, Tapia E, Santamaria J, et al. Mild hyperuricemia induces vasoconstriction and maintains glomerular hypertension in normal and remnant kidney rats. Kidney Int. 2005;67(1):237-247.
Clinical Interventions in Aging

\section{Publish your work in this journal}

Clinical Interventions in Aging is an international, peer-reviewed journal focusing on evidence-based reports on the value or lack thereof of treatments intended to prevent or delay the onset of maladaptive correlates of aging in human beings. This journal is indexed on PubMed Central, MedLine,

\section{Dovepress}

CAS, Scopus and the Elsevier Bibliographic databases. The manuscript management system is completely online and includes a very quick and fair peer-review system, which is all easy to use. Visit http://www.dovepress. com/testimonials.php to read real quotes from published authors. 